\title{
Acute Renal Failure and Renal Replacement Therapy in the Postoperative Period of Orthotopic Liver Transplant Patients Versus Nonelective Abdominal Surgery Patients
}

\author{
E. Biagioni, I. Cavazzuti, S. Busani, D. Trevisan, L. Zavatti, E. Ferrari, and G. Massimo
}

\begin{abstract}
Acute renal failure (ARF) often complicates the postoperative period of patients undergoing orthotopic liver transplantation (OLT); it is habitually associated with high mortality rates. Similarly, patients undergoing major nonelective abdominal surgery are prone to ARF because of their frequent preexistent morbidities, abdominal sepsis, and needed for extended surgical procedures. The aim of this study was to evaluate the incidence of ARF and use of renal replacement therapy (RRT) among OLT versus nonelective abdominal surgery patients and associations with clinical outcomes. We studied all the patients admitted to a surgical intensive care unit (ICU) from January 2008 to December 2009 after OLT or nonelective abdominal surgery. The inclusion criteria were an ICU stay of at least 48 hours and without prior end-stage renal failure. OLT patients $(n=84)$ were younger and less severly ill than surgery patients $(n=60)$. ARF occurrence was lower among the OLT $(29 \%)$ than the surgery group $(47 \%)$ requiring RRT in $71 \%$ and $53 \%$ of patients due to ARF, respectively. The ICU mortality of ARF patients in both groups (29\% OLT and 51\% surgery) were greater than among subjects without ARF (2\% and 6\%). The occurrence of ARF is common among these two patient groups, and associated with increased risk of death among in surgery $(+45 \%)$ versus in OLT $(+27 \%)$ patients.
\end{abstract}

\begin{abstract}
A CUTE RENAL FAILURE (ARF) is frequently observed in the postoperative period of patients undergoing orthotopic liver transplantation (OLT); reported incidences range from $12 \%$ to $70 \% .^{1}$ The development of ARF is closed related to medical and surgical complications. The mortality rate of OLT patients undergoing renal replacement therapy (RRT) may reach 90\%.2 Diabetes mellitus, a history of hepatorenal syndrome, massive intraoperative transfusions, long graft ischemia, and the use of nephrotoxic immunosuppressant agents and antibiotics seem to be the major causative factors involved in ARF appearance after OLT. ${ }^{1-4}$

As in OLT, renal dysfunction in patients undergoing emergency abdominal surgery is commonly associated with a high morbidity and mortality. It is generally related to hypoxic damage caused by systemic hypotension or reduced renal perfusion due to intra-abdominal hypertension. The preexisting risks factors, like mild to moderate renal dysfunction, diabetes mellitus, patient age over 65 years, and recent exposure to nephrotoxic drugs, are also important be considerations in the strat-
\end{abstract}

ification of renal failure management. ${ }^{5}$ The purpose of our study was to compare the incidence of ARF and the use of RRT among OLT patients versus those undergoing emergency abdominal surgery two populations at high risk for postoperative ARF.

\section{MATERIALS AND METHODS}

This retrospective analysis included all patients admitted to a surgical intensive care unit (ICU) from January 2008 to December 2009 after OLT or nonelective abdominal surgery. Inclusion criteria were an ICU stay of at least 48 hours and no prior end-stage renal failure. Age, SAPS (Simplified Acute Physiology Score) II score at the admission, occurrence of ARF according to the RIFLE (Risk, Injury, Failure, Loss and End-Stage

From the Surgical Intensive Care Unit, University Hospital of Modena, Modena, Italy.

Address reprint requests to Emanuela Biagioni, Surgical Intensive Care Unit, University Hospital of Modena, L.go del Pozzo 71, 41100 Modena, Italy. E-mail: emanuela545@katamail.com

0041-1345/-see front matter doi:10.1016/j.transproceed.2011.02.051 
Kidney) classification, ${ }^{6}$ use of RRT, ICU length of stay (LOS), and mortality data were collected for each patient. Statistical analysis used SPSS 15.0 software (SPSS Inc, Chicago, Ill, USA) with group comparisons performed by analysis of variance or chi-square tests A $P$ value $\leq .05$ was considered significant.

\section{RESULTS}

We studied 84 patients undergoing OLT and 60 admitted to ICU after emergency abdominal surgery. The OLT patients were younger $(56 \pm 9$ years $)$ and showed less severe SAPS II scores $(31 \pm 15)$ than the surgery group $(73 \pm 13$ years $[P<.01]$ and $48 \pm 16[P<.01])$. The $29 \%$ (24/84) incidence of ARF in the OLT group was lower $(P<.05)$ than in the surgery group $(29 / 60$ patients, $47 \%)$. The respective numbers who displayed ARF were 17 (71\%) and $15(53 \%)$. In both groups, SAPS II scores and ages of patients patients with versus without ARF were similar (Table 1). The ICU LOS in patients with ARF was longer after not significantly among the surgery

Table 1. Incidence of ARF and RRT, Age, SAPS II Score, ICU LOS, and ICU and Hospital Mortality in Patients Undergoing OLT and Emergency Abdominal Surgery

\begin{tabular}{|c|c|c|}
\hline & $\begin{array}{c}\text { OLT } \\
(n=84)\end{array}$ & $\begin{array}{l}\text { Surgery } \\
(n=60)\end{array}$ \\
\hline ARF, $\%$ ( $n / n$ total) & $29 \%(24 / 84)$ & $48 \%(29 / 60)$ \\
\hline RRT, \% ( $n / n$ total) & $71 \%(17 / 24)$ & $53 \%(17 / 29)$ \\
\hline \multicolumn{3}{|c|}{ Age, y $(\text { mean } \pm S D)^{*}$} \\
\hline Total & $56 \pm 9$ & $73 \pm 14$ \\
\hline ARF & $56 \pm 9$ & $70 \pm 15$ \\
\hline No ARF & $54 \pm 11$ & $76 \pm 11$ \\
\hline RRT & $54 \pm 13$ & $75 \pm 10$ \\
\hline \multicolumn{3}{|c|}{ SAPS II (mean $\pm \mathrm{SD})^{\star}$} \\
\hline Total & $31 \pm 15$ & $48 \pm 16$ \\
\hline No ARF & $27 \pm 14$ & $44 \pm 16$ \\
\hline ARF & $41 \pm 15$ & $51 \pm 15$ \\
\hline RRT & $43 \pm 17$ & $58 \pm 16$ \\
\hline \multicolumn{3}{|c|}{ ICU-LOS, d (mean \pm SD) } \\
\hline Total & $5 \pm 4$ & $11 \pm 24$ \\
\hline No ARF & $2 \pm 3$ & $6 \pm 5$ \\
\hline ARF & $8 \pm 6$ & $21 \pm 28$ \\
\hline RRT & $9 \pm 6$ & $11 \pm 6$ \\
\hline \multicolumn{3}{|c|}{ ICU mortality, $\%(n / n \text { total })^{\dagger}$} \\
\hline Total & $10 \%(8 / 84)$ & $28 \%(17 / 60)$ \\
\hline No ARF & $2 \%(1 / 60)$ & $6 \%(2 / 31)$ \\
\hline ARF & $29 \%(7 / 24)$ & $51 \%(15 / 29)$ \\
\hline RRT & $35 \%(6 / 17)$ & $53 \%(9 / 17)$ \\
\hline \multicolumn{3}{|c|}{ Hospital mortality, \% (n/n total) ${ }^{\dagger}$} \\
\hline Total & $11 \%(9 / 84)$ & $33 \%(20 / 60)$ \\
\hline No ARF & $2 \%(1 / 60)$ & $12 \%(4 / 31)$ \\
\hline ARF & $37 \%(8 / 24)$ & $55 \%(16 / 29)$ \\
\hline RRT & $41 \%(7 / 17)$ & $70 \%(12 / 17)$ \\
\hline
\end{tabular}

ARF, acute renal failure; RRT, renal replacement therapy; SAPS II; ICU, intensive care unit; LOS, length of stay; OLT, orthotopic liver transplantation; $\mathrm{SD}$, standard deviation.

${ }^{*} P<.05$ between OLT and surgery group

${ }^{\dagger} P<.05$ comparing ARF group with no ARF group.
( $21 \pm 28$ days) versus OLT ( $8 \pm 6$ days group). Similarly, the ICU and hospital mortality of surgery patients with $\operatorname{ARF}(51 \%$ and $55 \%$, respectively) were greater $(P>.05)$ than those of OLT patients with ARF (29\% and 33\%, respectively). The requirement for RRT increased in significantly the ICU and hospital mortality among ARF patients in both groups (Table 1).

\section{DISCUSSION}

This retrospective analysis indicated that during the postoperative period the occurrence of ARF was common among OLT patients ( $1 / 3$ of patients), but it was more common among subjects admitted to the ICU after nonelective major abdominal surgery ( $1 / 2$ patients). As expected, ARF occurrence was associated with an increased ICU mortality in both groups and an increased risk of death in surgery patients developing ARF (from $6 \%$ to $51 \%$, $+45 \%$ ), which was larger than that observed among OLT patients with ARF (from 2\% to 29\%, +27\%).

The occurrence of ARF and the clinical outcomes observed in our OLT patients were similar to those reported by other workers. ${ }^{1,2}$ The increased risk of death associated with the development of ARF indicated that renal impairment in the postoperative period, regardless of its primary cause and RRT treatment, are remarkable complications that must be carefully evaluated and managed by physicians. ${ }^{4,7}$ To our knowledge, few data are available on the relationship of postoperative renal dysfunction with clinical outcomes among patients undergoing nonelective major abdominal surgery. ${ }^{5}$ Our data clearly evidenced that ARF in this population, as in OLT patients, is a common complication in the postoperative period. Its appearance was closely related to bad patient outcomes, with a mortality among subjects developing ARF of about $50 \%$.

The differences in ARF incidence and mortality rate observed between OLT and surgery groups may be attributed to several factors. Patients in the surgery group were older and sicker than those in the OLT group. This observation can justify, at least in part, the higher rate of ARF and deaths observed in the former group. However, other important factors may play roles as, for instance, the effects of abdominal sepsis and its management, for example, fluids, antibiotics, and vasoactive drugs, on renal perfusion and tubular cell activities. ${ }^{8}$

In conclusion, our retrospective analysis indicated that ARF was a frequent, serious complication in the postoperative period of patients undergoing OLT or emergency abdominal surgery. However, in the latter group ARF seemed to occur more frequently than among OLT patients, with an absolute increased mortality risk of $45 \%$ compared to patients without ARF. 


\section{REFERENCES}

1. Nuno J, Cuervas-Mons V, Vicente E, et al: Renal failure after liver transplantation: analysis of risk factors in 139 liver transplant recipients. Transplant Proc 27:2319, 1995

2. Faenza S, Santoro A, Mancini E, et al: Acute renal failure requiring renal replacement therapy after orthotopic liver transplantation. Transplant Proc 38:1141, 2006

3. Crawford DHG, Endre ZH, Axelsen RA, et al: Universal occurrence of glomerular abnormalities in patients receiving liver transplants. Am J Kidney Dis 19:339, 1992

4. Platz KP, Mueller AR, Blumhardt G, et al: A comparison between cyclosporine and FK506. Transplantation 58:170, 1994
5. Levy EM, Viscoli CM, Horwitz RI, et al: The effect of acute renal failure on mortality: a cohort analysis. JAMA 275:1489, 1996

6. Kellum JA, Levin N, Bouman C, et al: Developing a consensus classification system for acute renal failure. Curr Opin Crit Care 8:509, 2002

7. Nair S, Verma S, Thuluvath P: Pretransplant renal function predicts survival in patients undergoing orthotopic liver transplantation. Hepatology 35:1179, 2002

8. Maerz L, Kaplan LJ: Abdominal compartment syndrome. Crit Care Med 36:S212, 2008 